\title{
First measurements of a scintillator based fast-ion loss detector near the ASDEX Upgrade divertor
}

Cite as: Rev. Sci. Instrum. 89, 101106 (2018); https://doi.org/10.1063/1.5038968

Submitted: 07 May 2018 . Accepted: 03 June 2018. Published Online: 07 August 2018

J. Gonzalez-Martin (D), J. Ayllon-Guerola (D), M. Garcia-Munoz (D), A. Herrmann, P. Leitenstern, P. De Marne, S. Zoletnik, A. Kovacsik, J. Galdon-Quiroga, J. Rivero-Rodriguez, M. Rodriguez-Ramos, L. Sanchis-Sanchez (D), J. Dominguez, ASDEX Upgrade Team, and MST1 Team

\section{COLLECTIONS}

Paper published as part of the special topic on Proceedings of the 22nd Topical Conference on High-Temperature Plasma Diagnostics

Note: Paper published as part of the Proceedings of the 22nd Topical Conference on High-Temperature Plasma Diagnostics, San Diego, California, April 2018.
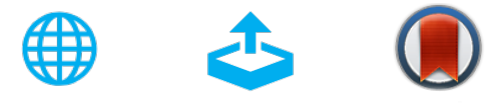

\section{ARTICLES YOU MAY BE INTERESTED IN}

A rotary and reciprocating scintillator based fast-ion loss detector for the MAST-U tokamak Review of Scientific Instruments 89, 101112 (2018); https://doi.org/10.1063/1.5039311

A fast feedback controlled magnetic drive for the ASDEX Upgrade fast-ion loss detectors Review of Scientific Instruments 87, 11 E705 (2016); https://doi.org/10.1063/1.4959913

High detection efficiency scintillating fiber detector for time-resolved measurement of triton burnup $14 \mathrm{MeV}$ neutron in deuterium plasma experiment

Review of Scientific Instruments 89, 101101 (2018); https://doi.org/10.1063/1.5032118

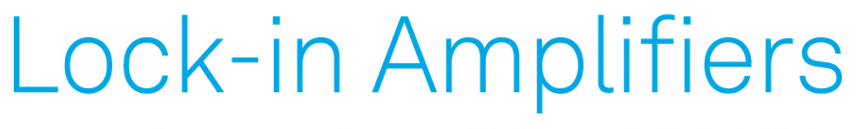
... and more, from DC to $600 \mathrm{MHz}$

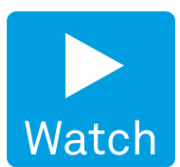




\title{
First measurements of a scintillator based fast-ion loss detector near the ASDEX Upgrade divertor
}

\author{
J. Gonzalez-Martin, ${ }^{1,2}$ J. Ayllon-Guerola, ${ }^{1,2}$ M. Garcia-Munoz, ${ }^{2,3}$ A. Herrmann, ${ }^{4}$ \\ P. Leitenstern, ${ }^{4}$ P. De Marne, ${ }^{4}$ S. Zoletnik, ${ }^{5}$ A. Kovacsik, ${ }^{5}$ J. Galdon-Quiroga, ${ }^{2,3}$ \\ J. Rivero-Rodriguez,, ${ }^{1,2}$ M. Rodriguez-Ramos, ${ }^{2,3}$ L. Sanchis-Sanchez, ${ }^{2,3}$ \\ J. Dominguez, ${ }^{1}$ ASDEX Upgrade Team, ${ }^{4, a)}$ and MST1 Team ${ }^{\text {b) }}$ \\ ${ }^{1}$ Department of Mechanical and Manufacturing Engineering, University of Seville, C/Camino de los \\ Descubrimientos $s / n$, Isla de la Cartuja, Seville, Spain \\ ${ }^{2}$ CNA (U. Sevilla, CSIC, J. de Andalucia), Seville, Spain \\ ${ }^{3}$ Department of Atomic, Molecular and Nuclear Physics, Faculty of Physics, University of Seville, \\ 41012 Seville, Spain \\ ${ }^{4}$ Max-Planck-Institut für Plasmaphysik, Garching, Germany \\ ${ }^{5}$ Wigner RCP, Budapest, Hungary
}

(Presented 16 April 2018; received 7 May 2018; accepted 3 June 2018; published online 7 August 2018)

\begin{abstract}
A new reciprocating scintillator based fast-ion loss detector has been installed a few centimeters above the outer divertor of the ASDEX Upgrade tokamak and between two of its lower Edge Localized Modes (ELM) mitigation coils. The detector head containing the scintillator screen, Faraday cup, calibration lamp, and collimator systems are installed on a motorized reciprocating system that can adjust its position via remote control in between plasma discharges. Orbit simulations are used to optimize the detector geometry and velocity-space coverage. The scintillator image is transferred to the light acquisition systems outside of the vacuum via a lens relay (embedded in a 3D-printed titanium holder) and an in-vacuum image guide. A charge coupled device camera, for high velocityspace resolution, and an $8 \times 8$ channel avalanche photo diode camera, for high temporal resolution (up to $2 \mathrm{MHz}$ ), are used as light acquisition systems. Initial results showing velocity-space of neutral beam injection prompt losses and fast-ion losses induced by a $(2,1)$ neoclassical tearing mode are presented. https://doi.org/10.1063/1.5038968
\end{abstract}

\section{INTRODUCTION}

In magnetically confined fusion plasmas, fast-ion confinement is essential to assure device integrity and heating efficiency, especially in ITER and future fusion reactors with a large population of fusion-born alpha particles. ${ }^{1}$ In present-day devices, scintillator-based Fast-Ion Loss Detectors (FILDs) ${ }^{2}$ are considered as one of the main diagnostics to study fast-ion loss mechanisms as they provide velocity-space measurements of escaping fast-ions with alfvénic temporal resolution.

Despite its unique capabilities, the fact that FILD can only provide local measurements makes a stand-alone FILD insufficient while trying to fully characterize the losses produced by 3D magnetohydrodynamic (MHD) perturbations. ${ }^{3}$ For that reason, different tokamaks have been equipped with more than one FILD, ${ }^{4-6}$ revealing the spatial dependency of fast-ion losses under different heating schemes, perturbative coil configurations, ${ }^{7}$ and MHD fluctuations. ${ }^{8}$ Particularly, the ASDEX Upgrade (AUG) tokamak counted so far with a set of three FILDs, all of them above the midplane. Aiming to improve the poloidal coverage of this set, a new detector (labeled FILD5) has recently been installed in between two

Note: Paper published as part of the Proceedings of the 22nd Topical Conference on High-Temperature Plasma Diagnostics, San Diego, California, April 2018.

a) See authors list in A. Kallenbach et al., Nucl. Fusion 53, 102015 (2017).

b) See authors list in H. Meyer et al., Nucl. Fusion 57, 102014 (2017). of the lower Edge Localized Modes (ELM) mitigation coils, providing direct measurements of the losses a few centimetres above the lower outer divertor, which opens the possibility to study x-point fluctuations on neutral beam injection (NBI) losses.

This document is organized as follows. A detailed description of the diagnostic setup [including probe head, velocityspace coverage, remotely controlled reciprocating system, optics relay, and data acquisition systems (DAQs)] is given in Sec. II. First measurements observed during the last experimental campaign (including NBI prompt losses and MHD induced fast-ion losses) are discussed in Sec. III. Section IV summarizes the document and briefs forthcoming efforts.

\section{DIAGNOSTIC SETUP}

The design of this new FILD is based on the three already existing detectors. Escaping ions reaching the wall on their gyro-orbits enter the detector head through a small slit impinging a scintillator-coated plate, inducing light. The slit, together with the protection head, collimates incoming ions, so only desired orbits strike the scintillator plate. The two-dimensional pattern of the emitted light depends on the gyroradii and pitchangle of the collimated trajectories while a short decay time (490 ns) of the scintillating material (TG-Green) enables to identify fast-ion avalanches produced by high-frequency MHD phenomena. 


\section{A. Detector head design and location}

The graphite cup (diameter $\sim 7 \mathrm{~cm}$ ) protecting sensitive components from heat and particle fluxes is D-shaped to maximize the number of fast-ions entering the scintillating chamber. The ion entrance aperture $(0.5 \mathrm{~mm}$ width, $2 \mathrm{~mm}$ long, and $10.5 \mathrm{~mm}$ deep) is placed $5 \mathrm{~mm}$ away from the scintillator plate, whose position within the light acquisition systems can be determined by lighting up a halogen lamp embedded on the head. To track the heat shield temperature, a thermocouple contacts the inner surface of the graphite cup. Additionally, a secondary plate has been implanted behind the scintillator plate, acting as a Faraday cup and allowing a complementary method for absolute measurements of fast-ion losses. A more detailed description of FILD probe heads can be found in Ref. 2.

To maintain the magnetic field lines lying on the scintillator plate and parallel to the collimator slit, the detector head is at $45^{\circ}$ with respect to the midplane and $12^{\circ}$ around its own axis. It rests between B-coils IL 3 and IL4 right above the divertor, as shown in Fig. 1. When FILD is not being used, the probe head's outermost surface remains sheltered by the two surrounding B-coil protection parts, which were milled to accommodate the diagnostic.

\section{B. Velocity-space coverage}

Full orbit simulations are used to optimize detector geometry and estimate velocity-space coverage depending on magnetic equilibrium. Particles with the gyroradius ranging from 2 to $8 \mathrm{~cm}$ and the pitch angle from $20^{\circ}$ to $80^{\circ}$ are initialized on the collimator slit. Trajectories are calculated backwards in time, identifying collisions with a 2D wall. Markers completing a total of three toroidal turns without colliding with

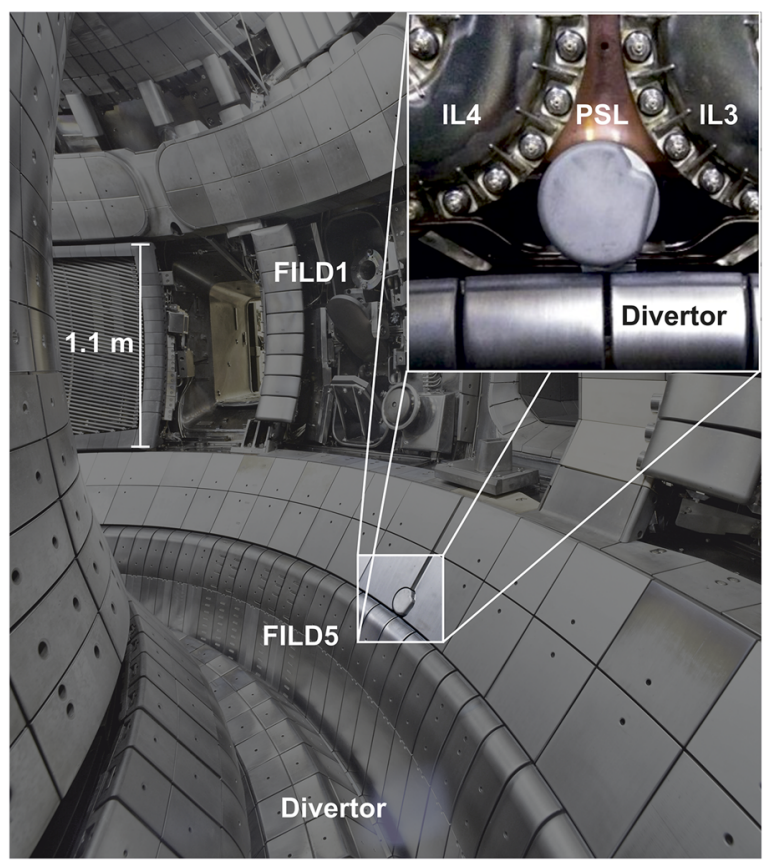

FIG. 1. ASDEX Upgrade vessel after FILD installation. The detector head can be spotted among the milled B-coil protection parts. The inset shows the FILD head during montage, surrounded by B-coils, divertor, and PSL. the wall are considered as covered by FILD. Velocity-space is calculated for different FILD insertions, enabling us to identify the optimal measuring position. Figure 2 shows the velocity-space coverage diagram calculated using the equilibrium reconstruction of a plasma discharge performed during the last campaign.

\section{FILD reciprocating system}

In order to expose the collimator slit to fast-ion orbits, movement from the out-vessel driving unit needs to be transmitted to the off-axis rod which holds the probe head (Fig. 3). For this reason, a rotary feedthrough is coupled to a wormgear by means of a 1-m double-cardan joint along a radial port originally dedicated to the B-coils. The worm-gear transmits its linear movement to the off-axis rod via two crank shafts (one on each side of the detector). The movement of the off-axis rod is restricted by a set of $4 \times 2$ bearings, which are clamped to a holder armed with two in-vessel end-ofstroke electrical switches. Signals provided by the switches are used by the Discharge Control System (DCS) to determine whether the programmed discharge is compatible with the current configuration of the FILDs.

The set of forefront mechanical elements is fastened to a metallic case which encloses part of the lens relay. Since this case is fixed to the PSL, electrical insulations made of vacuum-compatible polymers such as Polyether Ether Ketone $(\text { PEEK })^{9}$ and Vespel are used to contact components at vessel potential.

To reach its outermost position, FILD head needs to move $25 \mathrm{~mm}$ along its axis, which corresponds to a total of 20 turns of the rotary feedthrough. The rotation of this element is provided by the out-vessel driving unit ${ }^{10}$ (Fig. 4). It consists of a stepper motor (MICRON XTRUE 60), an absolute rotary encoder (PROFIBUS-DP class 2), a transmission belt, and a spring torque limiter. The torque limiter connects the stepper motor and the rotary encoder protecting the rotary feedthrough. Comparing the rotary encoder signal with the embedded motor encoder allows identifying slipping caused by in-vessel worm gear clogging. Both the motor and the rotary encoder are controlled by a Programmable Logic Computer (PLC) S7

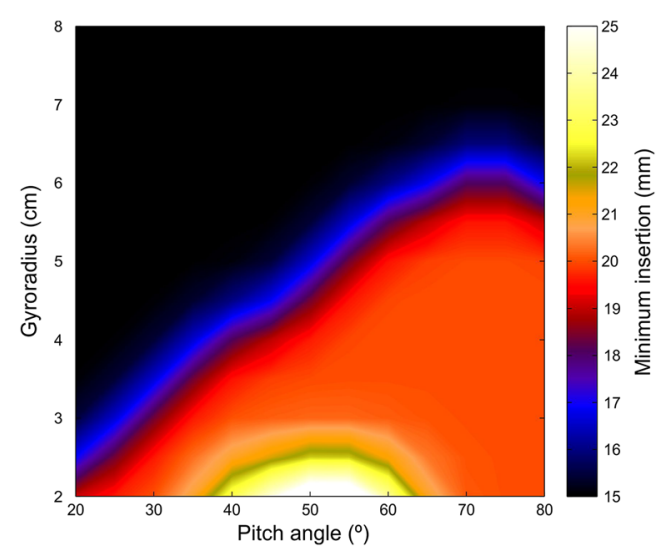

FIG. 2. Velocity-space coverage diagram for shot no. 34570 at $3.4 \mathrm{~s}$, showing full coverage for the FILD's outermost position. When FILD is inserted less than $15 \mathrm{~mm}$, coverage is zero since the collimator slit is behind the wall. 


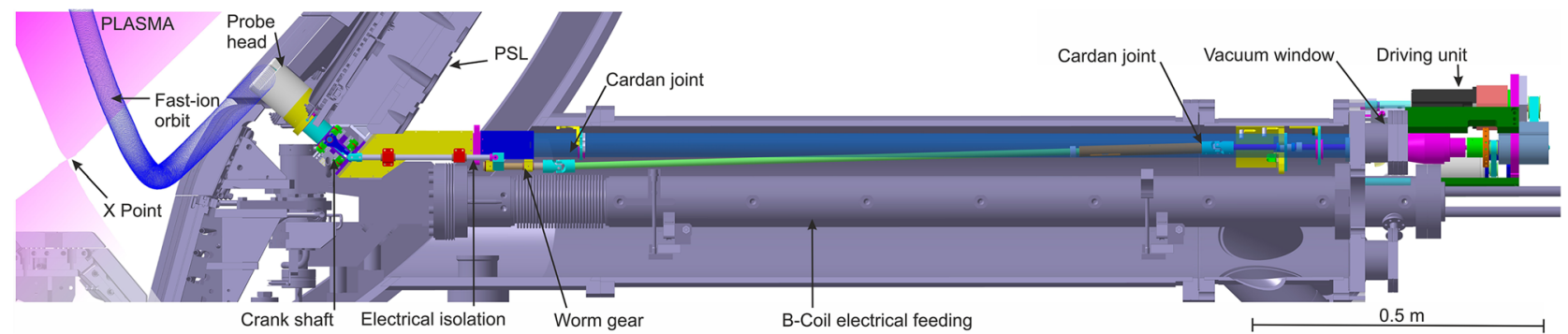

FIG. 3. Computer-aided design (CAD) view of the FILD system embedded on the AUG vessel. One can observe the in-vessel mechanical driving unit, including double-cardan joint, worm-gear, crank shaft, and bearing set. Additionally, the electrically isolated case containing the lens relay together with the pipe enclosing the image bundle is displayed.

SIMATIC. The most sensitive elements such as the main PLC and the motor controller are placed in the transfer room, so they are protected from the electric and magnetic fields that could harm them. The FILD operator can remotely communicate with the PLC using the human-machine interface system WinCC.

\section{Optics relay and light acquisition systems}

The light pattern emitted by the scintillating plate is transferred to the light acquisition systems outside of the vacuum via an in-vessel optics set. Four components can be distinguished in Fig. 5. First, a 3D printed titanium body occupies

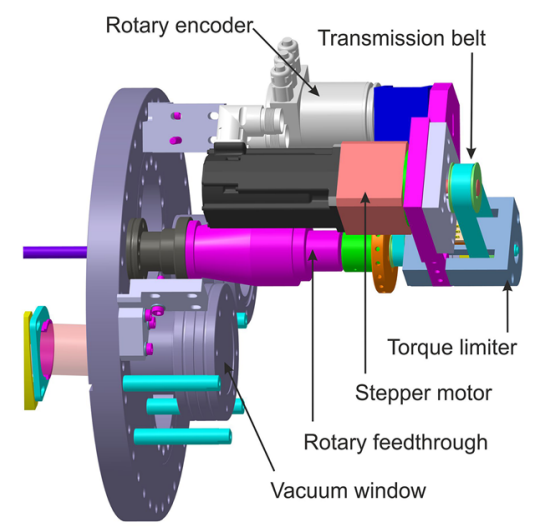

FIG. 4. Assembly of the different elements of the out-vessel remotely controlled driving unit (stepper motor, rotary encoder, transmission belt, and torque limiter). Additionally, the antireflection-coated vacuum window can be spotted next to the driving unit.

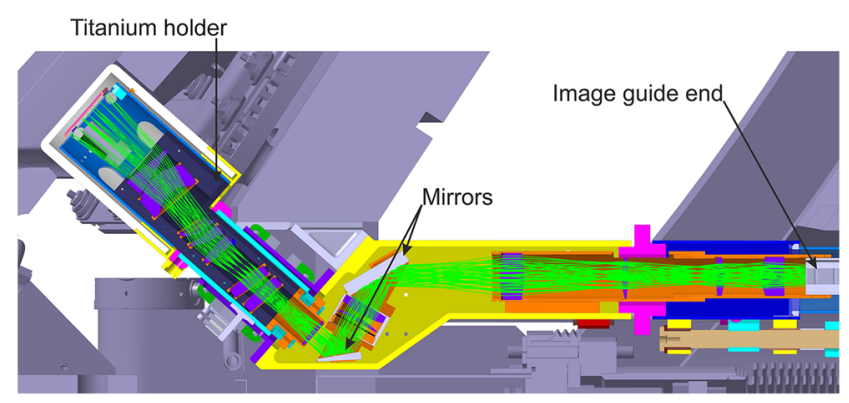

FIG. 5. FILD in-vessel optics. Green lines depict how the light coming from the scintillator rod is conducted toward the radial port and projected onto the image guide end. all the room from $4 \mathrm{~cm}$ to the scintillator plate until the end of the off-axis rod, holding a total of 8 lenses. This is needed to deviate the scintillating image through the off-axis rod. The titanium body was milled to accommodate the thermocouple case, calibration lamp, and Faraday cup wires. Second, already inside the PSL-clamped case, a set of 2 mirrors is used to deflect the light toward the radial port. Then, a set of 4 lenses held by an electrically insulated structure projects the light on an in-vessel coherent image guide. The image guide consists of $40 \times 50$ quartz-made optic fibers, whose transmission is not harmed by the neutron radiation. Finally, the light goes through an anti-reflection coated vacuum window which filters out light outside the bandwidth emitted by the scintillator.

The light beam coming from the vacuum window is diverted into two light acquisition systems via a drilled mirror which acts as an optimized beam splitter. The light acquisition systems are a Charge Coupled Device $(\mathrm{CCD})^{11}$ camera, for high velocity-space resolution, and an $8 \times 8$ channel Avalanche Photo Diode (APD) ${ }^{12}$ camera, for high temporal resolution (up to $2 \mathrm{MHz}$ ).

The APD camera replaces Photo Multiplier Tubes (PMTs) used in former FILDs. It can be directly installed near the vessel, saving the long fiber bundle needed by PMTs to carry the light from the torus hall to the transfer room. The detector chip of the APD camera is covered by an array of small lenses to focus all the incoming light into the sensitive regions of the detector. The APD camera communicates with a dedicated server in the control room via a 10 Gbit optic connection. Additionally, it receives an interlock trigger signal and a clock signal of $2 \mathrm{MHz}$.

The CCD camera $2 \mathrm{~m}$ Ethernet cable is insulated by a copper mesh against Ion Cyclotron Resonance Frequency (ICRF) cross talk. Its signal is sent to the control room via an optic wire using a data converter. Once in the control room, the signal is transferred to a Linux PC using a PCI card. When the DCS communicates in the beginning of the discharge via an interlock signal, the PC starts recording with a sample rate of 50 frames/s.

\section{FIRST MEASUREMENTS}

The new FILD has been operating during the last AUG experimental campaign. After measuring prompt and MHDinduced fast-ion losses in dedicated experiments, it was successfully used in other scenarios where FILDs normally 


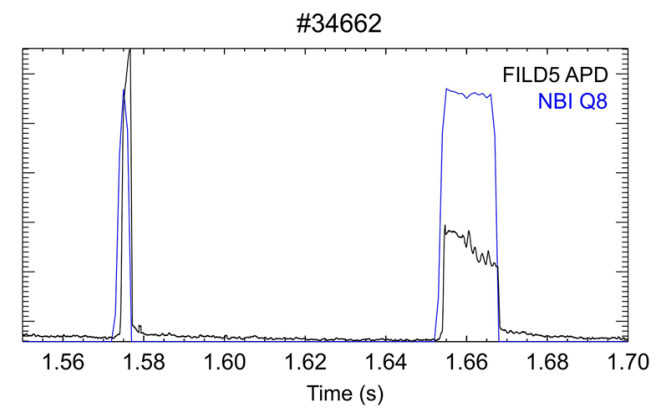

FIG. 6. Time trace of NBI Q8 applied power together with measured fast-ion losses with APD channel 8 corresponding to energy $\mathrm{E} \sim 93 \mathrm{keV}$ and pitch angle $\alpha \sim 45^{\circ}$.

have complications (e.g., upper single null). In this article, the most conspicuous results are shown as proof of principle, leaving the rest of the observations for forthcoming analysis.

\section{A. NBI prompt losses}

The ASDEX Upgrade tokamak has excellent qualities for fast particle experiments. Its heating system consists of $6 \mathrm{MW}$ of IRCH, $2 \mathrm{MW}$ of ECRH, and $20 \mathrm{MW}$ of NBI, distributed among 8 different sources at $60 / 93 \mathrm{keV}$ with different injection geometries, being the $93 \mathrm{keV}$ beams right above the new FILD.

Part of the NBI injected ions is born in unconfined orbits, depending on magnetic equilibrium, kinetic profiles, and applied beams; these particles (also known as prompt losses) are usually observed by FILD detectors.

Figure 6 shows the heating power time trace of NBI injector Q8 matching the scintillating light recorded by APD

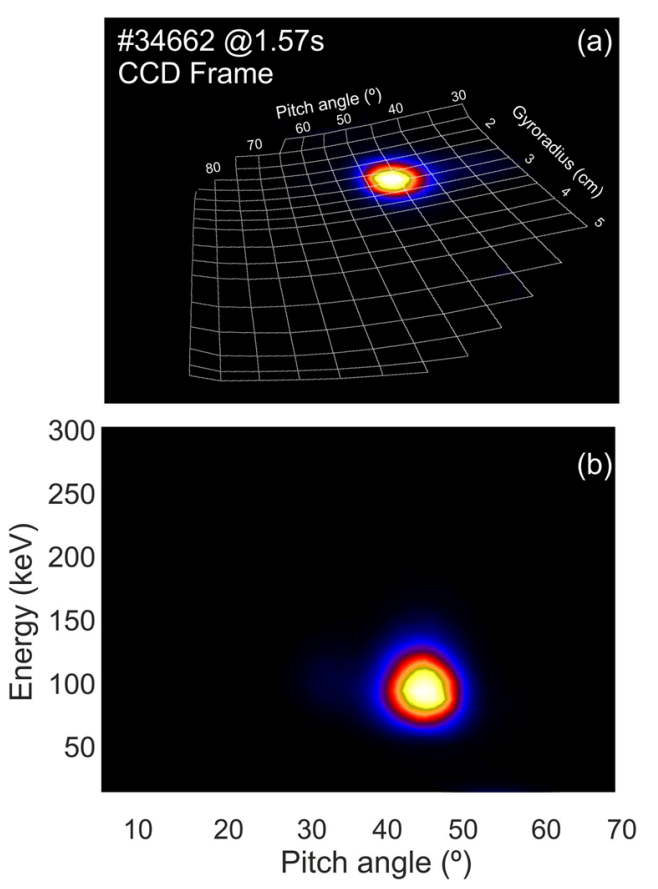

FIG. 7. (a) Calculated strike map overlayered onto the CCD frame during first NBI blip. (b) CCD frame triangulated according to strike map information and assuming deuterium ions.

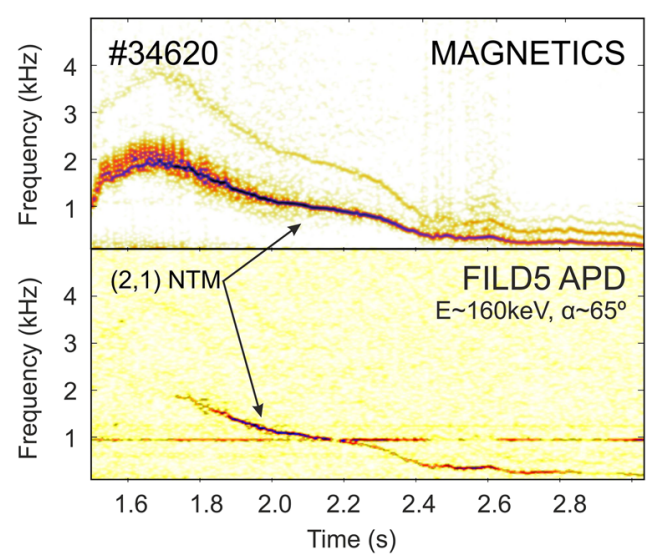

FIG. 8. Magnetic spectrogram showing a $(2,1)$ magnetic island (top). FILD APD channel 57 spectrogram (bottom). $(2,1)$ magnetic island is also observed in the APD spectrogram, indicating MHD induced fast-ion losses.

camera channel 8 during a discharge with a toroidal current of $I_{p}=0.45 \mathrm{MA}$ and a toroidal field of $B_{t}=2.4 \mathrm{~T}$. This confirms that the light emitted by the scintillator plate is produced by the fast-ion injected by the NBI source.

Besides, the velocity-space of the escaping ions can be inferred as follows. FILDSIM ${ }^{13}$ simulations are used to build a strike map containing the synthetic gyroradius and pitch angle distribution on the scintillator plate. The resulting strike map depends on the collimator slit dimensions and the magnetic field vector near the probe-head. The halogen lamp embedded in the graphite head is used to obtain a backlighted image of the scintillator plate which helps overlay the strike map on its correct position. The losses are observed to be centred around Larmor radii $\rho_{L} \sim 2.7 \mathrm{~cm}$ corresponding to deuterium ions injected at $93 \mathrm{keV}$ (given the magnetic field at FILD position $|\boldsymbol{B}|=2.3 \mathrm{~T}$ ). Moreover, the strike map is used for CCD frame triangulation to obtain the image shown in Fig. 7(b), allowing an easier comparison among experimental data and simulations.

\section{B. MHD induced fast-ion losses}

During 4 MW ICRH heated discharges, with a plasma current of $I_{p}=0.7 \mathrm{MA}$ and a toroidal magnetic field of $B_{t}=2.5 \mathrm{~T}$, a neoclassical tearing mode (NTM) evolves as shown in Fig. 8, magnetic spectrogram. The analysis of Mirnov coils' signals is used to calculate poloidal $(m=2)$ and toroidal $(n=1)$ mode numbers. Using electron cyclotron emission (ECE) fast measurements, the magnetic island is determined to be located at $\rho_{\text {pol }}=0.6$.

FILD APD camera adjacent channels show the same trace on the spectrogram during the saturated phase of the magnetic island, indicating coherent fast-ion losses produced by the NTM.

\section{CONCLUSIONS AND FUTURE WORK}

A new FILD has been designed, constructed, and commissioned near the ASDEX Upgrade lower divertor. Due to its location, innovative features have been included such as an off-axis reciprocating system, in-vessel movable lens relay, 
and coherent quartz image guide. PMTs have been replaced by an $8 \times 8$ channel APD camera for high temporal resolution measurements. During the last experimental campaign, NBI prompt losses and NTM induced fast-ion losses have been identified. This new detector improves the poloidal coverage of fast-ion losses on ASDEX Upgrade, which will be completed with the recently installed magnetically driven FILD4. ${ }^{14}$

\section{ACKNOWLEDGMENTS}

The authors would like to show their gratitude to Wolfgang Popken for his contribution to this project.

This work has been carried out within the framework of the EUROfusion Consortium and has received funding from the Euratom research and training programme 2014-2018 under Grant Agreement No. 633053. The views and opinions expressed herein do not necessarily reflect those of the European Commission.
${ }^{1}$ A. Fasoli et al., Nucl. Fusion 47, S264 (2007).

${ }^{2}$ M. Garcia-Munoz, H.-U. Fahrbach, and H. Zohm, Rev. Sci. Instrum. 80, 053503 (2009).

${ }^{3}$ M. Garcia-Munoz et al., Plasma Phys. Controlled Fusion 55, 124014 (2013).

${ }^{4}$ X. Chen et al., Rev. Sci. Instrum. 83, 10 D707 (2012).

${ }^{5}$ S. Baeumel et al., Rev. Sci. Instrum. 75, 3563 (2004).

${ }^{6}$ S. J. Zweben, R. L. Boivin, M. Diesso, S. Hayes, H. W. Hendel, H. Park, and J. D. Strachan, Nucl. Fusion 30, 1551 (1990).

${ }^{7}$ M. A. Van Zeeland et al., Plasma Phys. Controlled Fusion 56, 015009 (2014).

${ }^{8} \mathrm{~S}$. J. Zween, et al., 'Recent progress on MHD-induced loss of D-D fusion products in TFTR'. (PPPL-2934) (1993).

${ }^{9}$ I. Zammuto, M. Rott, B. Streibl, W. Suttrop, and T. Vierle, Fusion Eng. Des. 86, 1067 (2011).

${ }^{10}$ P. de Marné, A. Herrmann, and P. Leitenstern, Fusion Eng. Des. 123, 754 (2017).

${ }^{11}$ G. Náfrádi, A. Kovácsik, G. Pór, M. Lampert, Y. Un-Nam, and S. Zoletnik, Nucl. Instrum. Methods Phys. Res., Sect. A 770, 21 (2015).

${ }^{12}$ E. Dunai, S. Zoletnik, J. Sárzközi, and A. R. Field, Rev. Sci. Instrum. 81, 103503 (2010).

${ }^{13} \mathrm{~J}$. Galdon-Quiroga et al., "Velocity-space sensitivity and tomography of scintillator-based fast-ion loss detectors," Plasma Phys. Controlled Fusion (submitted).

${ }^{14}$ J. Ayllon et al., Rev. Sci. Instrum. 87, $11 \mathrm{E} 705$ (2016). 